\title{
Calculation Method for Visibility of Emergency Sign in Fire Taking into Account of Smoke Adhesion
}

\author{
AKIZUKI YUKI ${ }^{1}$, TANAKA TAKEYOSHI ${ }^{1}$, SUZUKI HIDEKAZU ${ }^{1}$, and \\ TSUCHIHASHI TSUNETO ${ }^{2}$ \\ ${ }^{1}$ Disaster Prevention Research Institute, Kyoto University \\ Gokasho, Uji, Kyoto 611-0011, Japan \\ ${ }^{2}$ Building Physics Department, General Building Research Corporation \\ 5-8-1, Fujishirodai, Suita, Osaka 565-0873, Japan
}

\section{ABSTRACT}

In making a plan for safe evacuation under smoke in fire, visibility of evacuation signs is of crucial significance. However, there have been very few studies on the calculation method for the luminous stimuli in fire smoke, thereby leaving effective evacuation methodology unattained. In fire situation, we have to consider factors affecting sign's visibility such as power failure, obscuration by smokes and smoke adhesion to the sign surface or light sources. In order for emergency signs to function properly for evacuation, we need to assure the sufficient visibility in every situation. Therefore it is necessary to establish a calculation method of luminous stimuli in fire smoke to evaluate evacuee's visibility quantitatively. This paper proposes a calculation model of target luminance in fire-smoke taking into account of smoke adhesion. Practical application is also presented.

KEYWORDS: smoke, evacuation, visibility, luminance, adhesion

\section{NOMENCLATURE LISTING}

\begin{tabular}{|c|c|c|c|}
\hline$A_{\text {air }}$ & $\begin{array}{l}\text { surface area of lower air layer } \\
\mathrm{m}^{2}\end{array}$ & $A_{\text {interface }}$ & $\begin{array}{l}\text { interface area between upper smoke } \\
\text { layer and lower air layer } \mathrm{m}^{2}\end{array}$ \\
\hline$A_{\text {smoke }}$ & $\begin{array}{l}\text { surface area of upper smoke } \\
\text { layer } \mathrm{m}^{2}\end{array}$ & $A_{i}$ & $\begin{array}{l}\text { area of wall element in upper smoke } \\
\text { layer } \mathrm{m}^{2}\end{array}$ \\
\hline$A_{j}$ & $\begin{array}{l}\text { area of wall element in lower air } \\
\text { layer } \mathrm{m}^{2}\end{array}$ & $C_{s}$ & optical smoke density 1/m \\
\hline$D$ & $\begin{array}{l}\text { visual range between target and } \\
\text { human eye m }\end{array}$ & $D_{(i)}$ & $\begin{array}{l}\text { visual range through upper smoke } \\
\text { layer m }\end{array}$ \\
\hline$F_{\text {air }}$ & $\begin{array}{l}\text { illuminance intensity in lower } \\
\text { air layer lm }\end{array}$ & $D_{(j)}$ & $\begin{array}{l}\text { visual range through lower air layer } \\
\mathrm{m}\end{array}$ \\
\hline$F_{\text {smoke }}$ & $\begin{array}{l}\text { illuminance intensity in upper } \\
\text { smoke layer lm }\end{array}$ & $k$ & scattering ratio of $C_{s}(-)$ \\
\hline$K_{a}$ & absorption coefficient 1/m & $K_{s}$ & scattering coefficient 1/m \\
\hline$I$ & specific intensity cd & $L_{0}$ & initial luminance $\mathrm{cd} / \mathrm{m}^{2}$ \\
\hline${ }_{a} L_{D(i)}$ & $\begin{array}{l}\text { target luminance in upper } \\
\text { smoke layer } \mathrm{cd} / \mathrm{m}^{2}\end{array}$ & $L_{1}, L_{2}$ & $\begin{array}{l}\text { transmitting luminance though } \\
\text { glass/acrylic panels } \mathrm{cd} / \mathrm{m}^{2}\end{array}$ \\
\hline$L_{D(j)}$ & $\begin{array}{l}\text { target luminance in lower air } \\
\text { layer } \mathrm{cd} / \mathrm{m}^{2}\end{array}$ & $L_{t}$ & $\begin{array}{l}\text { transmitting luminance through } \\
\text { smoke } \mathrm{cd} / \mathrm{m}^{2}\end{array}$ \\
\hline$L_{\text {air }}$ & $\begin{array}{l}\text { luminance scattering in lower } \\
\text { air layer } \mathrm{cd} / \mathrm{m}^{2}\end{array}$ & $L_{e}$ & $\begin{array}{l}\text { light source luminance of sign } \\
\mathrm{cd} / \mathrm{m}^{2}\end{array}$ \\
\hline$L_{\text {smoke }}$ & $\begin{array}{l}\text { luminance scattering in upper } \\
\text { smoke layer } \mathrm{cd} / \mathrm{m}^{2}\end{array}$ & $\Delta T$ & temperature rise (K) \\
\hline \multicolumn{4}{|l|}{ Greek } \\
\hline$\zeta$ & $\begin{array}{l}\text { luminance reduction rate due to } \\
\text { smoke adhesion (-) }\end{array}$ & $\eta$ & adhesion coefficient m/s \\
\hline
\end{tabular}




$\begin{array}{clcl}{ }_{0} \rho & \text { iInitial reflectance (-) } & { }_{a} \rho & \text { smoke-adhered reflectance (-) } \\ \rho_{e} & \text { reflectance of sign (-) } & \rho_{\text {smoke }} & \text { reflectance of smoke particles (-) } \\ \rho_{i} & \begin{array}{l}\text { reflectance of wall element in } \\ \text { upper smoke layer (-) }\end{array} & \rho_{j} & \begin{array}{l}\text { reflectance of wall element in lower } \\ \text { air layer (-) }\end{array} \\ { }_{0} \tau & \text { initial transmittance (-) } & { }_{a} \tau & \text { smoke-adhered transmittance (-) }\end{array}$

$\tau_{e} \quad$ transmittance of sign (-)

\section{INTRODUCTION}

It is widely recognized that three factors are important to properly design visual environment [1], i.e., environmental conditions, visual object's conditions and human visual ability. The first two factors determine visual stimulus, and the last one determines visual sensitivity. Visual response and evaluation, i.e., visibility, depends on both visual stimulus and visual sensitivity. Visual stimulus is represented by four physical elements; size of a visual target, background luminance, luminance contrast between target and background, and viewing time. If the viewing time is more than $100 \mathrm{~ms}$, the visibility becomes stable regardless of time [2]. Therefore, we use only three physical elements without viewing time to calculate visibility. Human visual ability consists of many functions like view field and color sensitivity and so on, but usually the most important is visual acuity. There is a significant difference of visual acuity not only among age groups but also among individuals [3]. Akizuki et al. proposes the evaluation method for visibility [1,3]. By using this, we can estimate varied visual environments to assign the values of three physical elements and visual acuity.

In fire situation, we have to consider additional factors affecting visibility; power failure and obscuration by smoke. In order for emergency signs to function properly for evacuation, we need to secure enough visibility in every situation, therefore it is necessary to establish a calculation method of luminous stimuli in fire smoke to evaluate evacuee's visibility quantitatively [4]. Under a fire, we have to treat the effects of absorption and scattering of smoke on luminous stimuli: the background luminance, target luminance and luminance contrast. Another factor to be considered is that smoke emitted by the fire adheres to the sign surface, light sources, and surrounding walls, causing deterioration of transmission ratio and insufficient light.

Therefore, all of them need to be incorporated into the calculation of sign's visibility. This paper proposes a calculation model of target luminance in fire-smoke taking into account of smoke adhesion. Also, practical application is presented using cases.

\section{PREDICTION MODEL OF VISUAL STIMULUS IN FIRE SMOKE}

\section{Basic Model of Optical Transmission Through Scattering Medium [5,6]}

In this section, we construct the basic model of optical transmission through scattering medium according to Chandrasekhar [5] and Matsuura [6]. Equation 1 is the well-known law of Lambert-Beer [5]:

$I_{s}=I_{o} \times e^{-C s D}$

where $I_{o}$ is the incident light flux, $I_{s}$ is the transmitted light flux.

$C_{s}$ consists of absorption coefficient $K_{a}$ and scattering coefficient $K_{s}$, i.e., 
$C_{s}=K_{a}+K_{s}$

Jin [7] measured the scattering ratio $k\left(=K_{s} / C_{s}\right)$ of various types of smoke, and obtained the value as follows:

$k=$ const. $=\left\{\begin{array}{l}0.5(\text { smoldering fire smoke }) \\ 1.0(\text { flaming fire smoke })\end{array}\right.$

Matsuura rearranged the basic model of optical transmission with scattering and absorbing medium based on Chandrasekhar's Eq. 6. Figure 1 shows the concept of the model in a closed space.

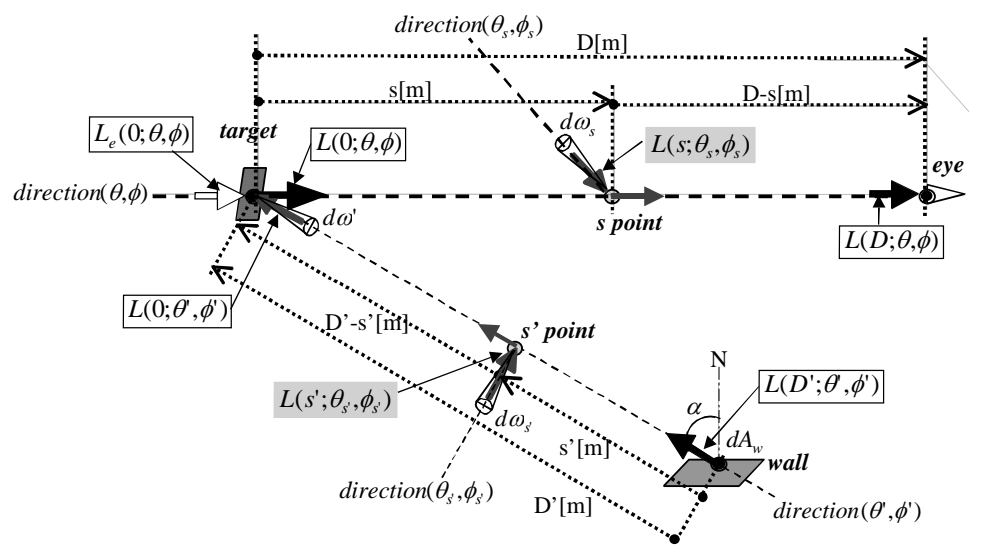

Fig. 1. Concept of the optical transmission model in a closed space [6].

The luminance $L(D ; \theta, \phi)$ at observer's eye, which is located in direction $(\theta, \phi)$ at distance $\mathrm{D}$ from the target, is given as follows:

$L(D ; \theta, \phi)=L(0 ; \theta, \phi) e^{-C_{s} D}+\int_{0}^{D} k C_{s}\left\{\int_{0}^{4 \pi} P\left(\theta, \phi: \theta_{s}, \phi_{s}\right) L\left(s ; \theta_{s}, \phi_{s}\right) \frac{d \omega_{s}}{4 \pi}\right\} e^{-C_{s}(D-s)} d s$

where $\left(\theta_{s}, \phi_{s}\right)$ is the incidence angle while $(\theta, \phi)$ is the directional angle of emission. $d \omega_{s}$ is the solid angle in the direction of $\left(\theta_{s}, \phi_{s}\right) . P\left(\theta, \phi: \theta_{s}, \phi_{s}\right)$ is called the phase function of which value depends on properties of scattering medium, and if the medium is isotropic scatter, the phase function is equal to 1 . In general, the phase function satisfies:

$\frac{1}{4 \pi} \int_{0}^{4 \pi} P\left(\theta, \phi: \theta_{s}, \phi_{s}\right) d \omega_{s}=1$

Here the first term of Eq. 4 means the extinction of the luminance $L(0 ; \theta, \phi)$ during the travel in the medium with optical smoke density $C_{s}$ to the observation point located at distance $\mathrm{D}$ from the target. The second term of Eq. 4 shows the sum of the scattering luminance $L\left(s ; \theta_{s}, \phi_{s}\right)$, which is incident from direction $\left(\theta_{s}, \phi_{s}\right)$ and scattered in direction $(\theta, \phi)$ at an arbitrary point " $\mathrm{s}$ ", from $\mathrm{s}=0$ (target) to $\mathrm{s}=\mathrm{D}$ (observer's eye). If 
$L\left(s ; \theta_{s}, \phi_{s}\right)$ is assumed to constant regardless of the position or the direction, the basic model of the optical transmission through a scattering medium is expressed as follows:

$$
L(D ; \theta, \phi)=L(0 ; \theta, \phi) e^{-C_{s} D}+k L(\theta, \phi)\left(1-e^{-C_{s} D}\right)
$$

where $L(\theta, \phi)$ means the sum of the scattering luminance in direction $(\theta, \phi)$ from the distance between target and observer's eye.

We consider that the surface luminance of the target, $L(0 ; \theta, \phi)$, consists of two portion; the emitting luminance for itself $L_{e}(0 ; \theta, \phi)$ and the sum of reflected luminance surrounding the target, i.e.,

$$
L(0 ; \theta, \phi)=L_{e}(0 ; \theta, \phi)+\int_{0}^{2 \pi} \rho\left(x ; \theta, \phi: \theta^{\prime}, \phi^{\prime}\right) L\left(0 ; \theta^{\prime}, \phi^{\prime}\right) d \omega^{\prime}
$$

where

$$
d \omega^{\prime}=\frac{\left(\cos \alpha \times d A_{w}\right)}{D^{\prime 2}}
$$

$L\left(0 ; \theta^{\prime}, \phi^{\prime}\right)=L\left(D^{\prime} ; \theta^{\prime}, \phi^{\prime}\right) e^{-C_{s} D^{\prime}}+k L\left(\theta^{\prime}, \phi^{\prime}\right)\left(1-e^{-C_{s} D^{\prime}}\right)$

where $\rho\left(0 ; \theta, \phi: \theta^{\prime}, \phi^{\prime}\right)$ is the reflectance function of the target surface. $L\left(0 ; \theta^{\prime}, \phi^{\prime}\right)$ is the reflected luminance that entered the target of solid angle $d \omega^{\prime}$ in direction $\left(\theta^{\prime}, \phi^{\prime}\right) . D^{\prime}$ is the distance between the target and an arbitrary point on the wall surface. $L\left(\theta^{\prime}, \phi^{\prime}\right)$ means the sum of the scattering luminance in direction $\left(\theta^{\prime}, \phi^{\prime}\right)$ from the distance between target and an arbitrary point of wall, where $L\left(s^{\prime} ; \theta_{s^{\prime}}, \phi_{s^{\prime}}\right)$ is assumed to be constant regardless of the position or the direction.

The optical transmission model through scattering medium in a close space can be obtained by Equations from Eq. 6 to Eq. 9. We can calculate the luminance at observer's eye, $L(D ; \theta, \phi)$, from three kinds of luminance; the emitting luminance of target $L_{e}(0 ; \theta, \phi)$, the reflected luminance by an arbitrary point on the wall $L\left(D^{\prime} ; \theta^{\prime}, \phi^{\prime}\right)$, and the scattering luminance by $L(\theta, \phi)$ and $L\left(\theta^{\prime}, \phi^{\prime}\right)$.

\section{Approximation of the Optical Transmission Model [6]}

If the reflection luminance by an arbitrary point on the wall $L\left(D^{\prime} ; \theta^{\prime}, \phi^{\prime}\right)$ is constant $L_{\text {space }}$, Eq. 7 can be reduced to Eq. 10.

$L(0 ; \theta, \phi)=L_{e}(0 ; \theta, \phi)+L_{\text {space }}$

Furthermore, if the luminance distribution of scattering medium, i.e., $L(\theta, \phi)$ and $L\left(\theta^{\prime}, \phi^{\prime}\right)$, is uniform, the scattering luminance is constant at any position and in any direction, and can be considered as the same as $L_{\text {space }}$. Then we can approximate Eq. 6 as follows: 
$L(D ; \theta, \phi)=L_{e}(0 ; \theta, \phi) e^{-C_{s} D}+L_{\text {space }}\left\{e^{-C_{s} D}+k\left(1-e^{-C_{s} D}\right)\right\}$

\section{Introducing Smoke Adhesion}

The variables affected by smoke adhesion are the emitting luminance of target and the reflection luminance in the closed space (i.e., the sum of illuminance intensity in the space and medium reflectance surface of the space). It is considered that the thicker the smoke concentration, the more the adhesion, although there is no previous study to quantify it in realistic fire configurations. Therefore in the following section we examine how to estimate the adhesion according to the stages of a fire.

\section{EXPERIMENT ON SMOKE ADHESION}

In order to investigate the effects of smoke adhesion on luminous stimuli, we conducted an experiment to measure the smoke density and reduction of transmittance by smoke adhesion.

\section{Methods}

\section{Experimental Apparatus}

As shown in Fig. 2, the apparatus consists of three parts; fire room, luminous target, and luminous measurement instrument. The size of the fire room is $00.8 \times H 0.8 \times W m$. The width $(W)$ of the fire room is adjustable by $0.25 \mathrm{~m}$ from $0.75 \mathrm{~m}$ to $2.0 \mathrm{~m}$. The fire room is made of calcium silicate board. The reflectance of board surface is 0.038 . At the Side-B, there are nine glass/acrylic panels not only to remove one by one with a given time interval for measuring various smoke-adhered transmittance, but to explore the effects of material on smoke adhesion. The glass of Side-A wasn't removed throughout the experiment. The initial transmittance of glass and acrylic panel were found to be ${ }_{0} \tau_{\text {glass }}=0.923$ and ${ }_{0} \tau_{\text {acrylic }}=0.951$, respectively.

In order to monitor the temperature distribution, 15 thermocouples are set vertically by $0.05 \mathrm{~m}$ intervals at the corner of Side-B in the fire room. The light source of the luminous target is an incandescent lamp $(100 \mathrm{~W}, 750 \mathrm{~lm})$. The luminous target is a circular hole of $0.15 \mathrm{~m}$ diameter in a wall of the integrating sphere, and it is centrally located at Side-A. A luminance meter (MINOLTA LS-110) is set at Side-B on the centerline of the luminous target.

\section{Fire Source}

We use three types of fire sources; kerosene $50 \mathrm{ml}$, cotton $150 \mathrm{~g}$, and soft-polyurethane $50 \mathrm{~g}$. These emit two kinds of smoke; black smoke from flaming kerosene and softpolyurethane, and white smoke from smoldering cotton wicks. The fire source is placed at the center on the floor of the fire room. Soft-polyurethane is ignited by applying kerosene $5.0 \mathrm{ml}$.

\section{Procedure}

In the experiment, we first let smoke fill up the fire room until the fuel is exhausted when the optical smoke density $C_{s}$ reaches the maximum. Then we measure the light intensity and $C_{s}$ continuously, while the smoke is diluted till $C_{s}=0$ due to mechanical exhaust or natural ventilation. The velocity of mechanical ventilation (diameter $\phi=0.10 \mathrm{~m}$ ) is 


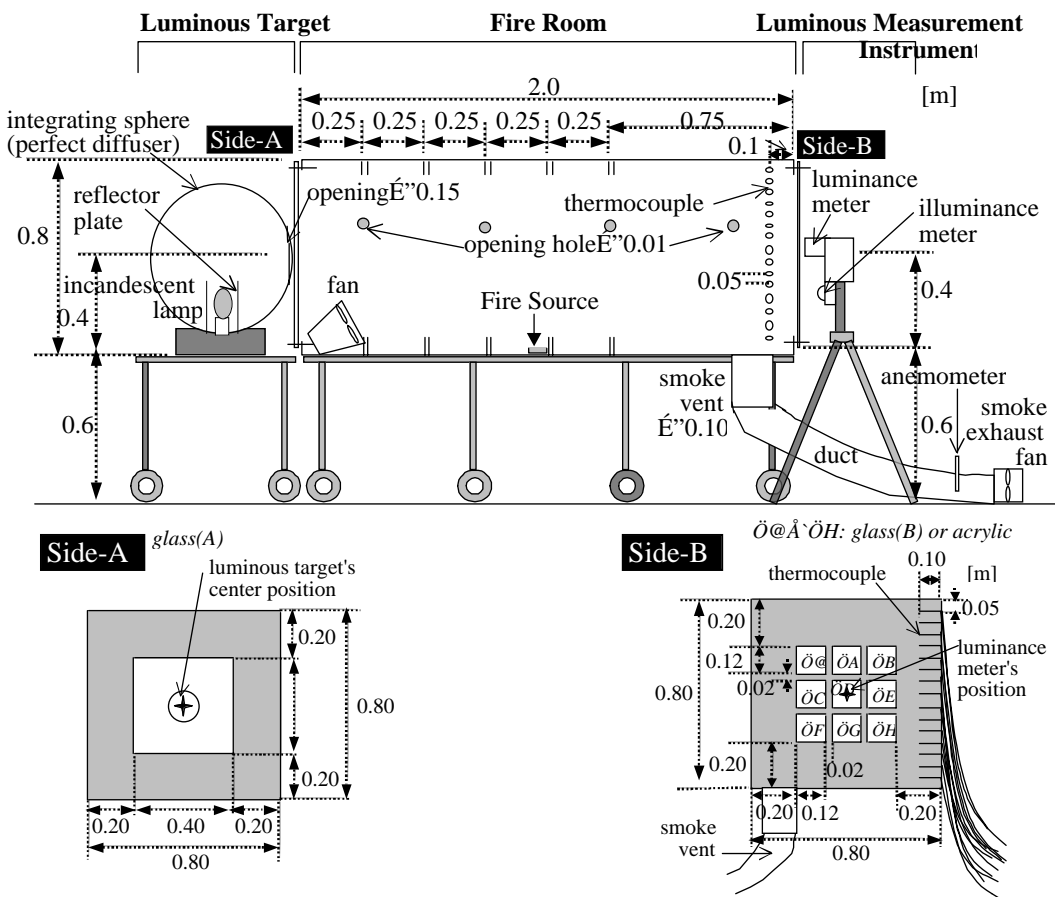

Fig. 2. Experimental apparatus.

set at three conditions: $0,1.0$ and $2.0 \mathrm{~m} / \mathrm{s}$. The smoke is continuously stirred by a fan set inside the fire room to achieve homogeneous distribution of smoke. After a certain time elapsed, nine glass/acrylic panels in Side-B are removed one by one with a given time interval, and the transmittance ${ }_{a} \tau$ of each smoke-adhered glass/acrylic is measured. The numbers of tests of kerosene cotton and soft-polyurethane are four, five and two, respectively.

\section{Apparatus Performance}

Figure 3 shows the distribution of temperature rise $\Delta T$ in the fire room. $\Delta T_{h=0.05}$ denotes the temperature rise at height $0.05 \mathrm{~m}$ and so on. There are slight differences among the temperatures at different heights, $\Delta T_{h=0.05}$ through $\Delta T_{h=0.75}$ due to effectively mixing of smoke by fan, i.e., stratification into smoke layer was not observed in fire room.

Figure 4 shows the distribution of transmittance ${ }_{a} \tau$ at the nine parts of the glass at SideA of which positions correspond to those of nine glasses of Side-B. The numbers in Fig. 4 show differences of ${ }_{a} \tau$ between at each position and the average, ${ }_{a} \tau_{i}-{ }_{a} \tau_{\text {average }}$, on a panel. The difference of ${ }_{a} \tau$ among those positions is trivial $( \pm 0.06)$, so it can be considered that the spatial distribution of ${ }_{a} \tau$ is uniform. Therefore the difference of the adhesion among the positions is considered to be negligible. 


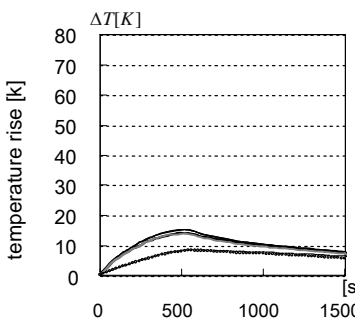

(1)Cotton

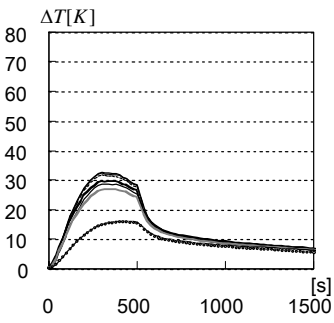

(2)Kerosene

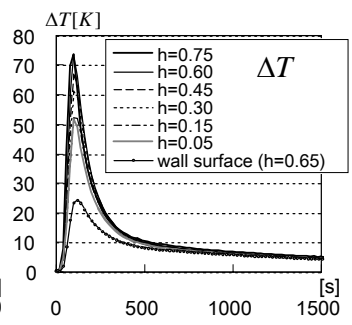

(3)Soft-Polyurethane

Fig. 3. Temperature distribution in the fire room (natural vent condition).

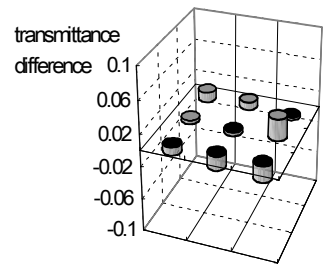

(1)Cotton

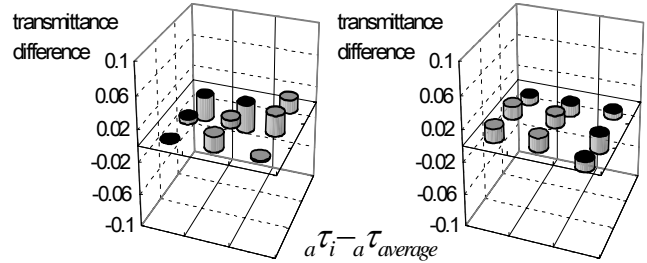

(2)Kerosene

(3)Soft-Polyurethane

Fig. 4. ${ }_{a} \tau$ Distribution among positions (natural vent condition).

\section{Luminance Reduction Rate Due to Smoke Adhesion $\zeta$}

\section{Definition of $\zeta$}

In this research, the luminance reduction rate due to smoke adhesion $\zeta$ is calculated by the smoke-adhered transmittance ${ }_{a} \tau\left({ }_{a} \tau \leq_{0} \tau\right)$ of smoke-adhered glass/acrylic panels. The initial transmittance of glass/acrylic panels ${ }_{0} \tau\left(0<_{0} \tau \leq 1\right)$, and the smoke-adhered transmittance ${ }_{a} \tau$ are given by

${ }_{0} \tau=\frac{L_{1}}{L_{0}} \quad$ and $\quad{ }_{a} \tau=\frac{L_{2}}{L_{0}}$

Respectively, where $L_{0}$ is the initial luminance, and $L_{1}$ and $L_{2}$ are the transmitting luminance through glass/acrylic panels in Fig. 5. Finally, the luminance reduction rate due to smoke adhesion $\zeta$ can be obtained as

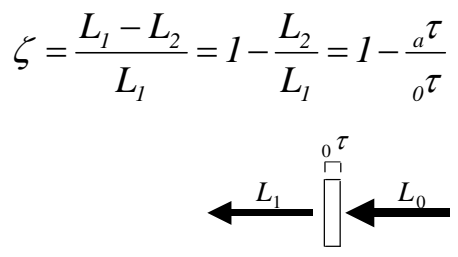

(1) Clean panel

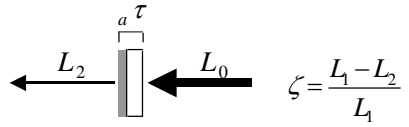

(2) Smoke-adhered panel

Fig. 5. Concept of $\zeta$. 
Considering the emergency signs, we examined two materials, i.e., glass (for light sources) and acrylic plate (for emergency). Figure 6 shows comparison of $\zeta$ between glass and acrylic panels. $\zeta$ of acrylic tends to be slightly higher than $\zeta$ of glass, but on the whole, it can be concluded that it is not necessary to concern about the effects of the material on smoke adhesion. Rather, fire source affects much more the value of $\zeta$.

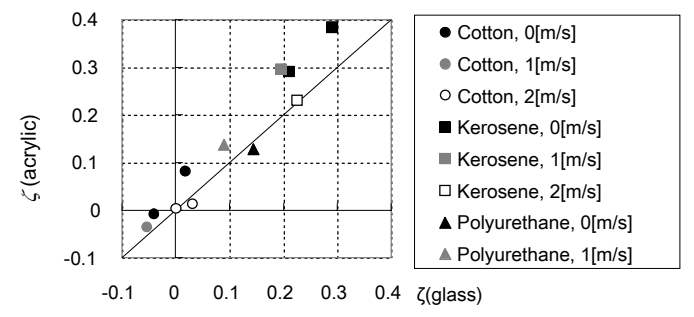

Fig. 6. Comparison of $\zeta$ among materials to adhere per fire source and vent velocity.

\section{Relations between Luminance Reduction Rate due to Smoke Adhesion $\zeta$ and Smoke Density $C_{s}$}

The amount of smoke adhesion is expected to be a function of exposure time and smoke density. Optical smoke density meters are also subjected to adhesion of smoke themselves, it is impossible to measure exact density unless adhered smoke is not kept clean. Considering the adhesion to apparatus of this experiment, smoke particles adhere both glasses of Side-A and Side-B. Therefore, we incorporated $\zeta$ into Lambert-Beer's law as shown by Eq. 14 to calculate the true value of $C_{s}$ in the fire room. Using, the smoke density is calculated according to attenuation of light intensity.

$C_{s}=-\frac{1}{D} \log _{e} \frac{L_{t}}{L_{0}(1-\zeta)^{2}}$

Figure 7 shows one of the results of $\zeta, C_{s}$ without adhesion, $\log _{10} L_{t}$ with adhesion, the temperature rise of the fire room $\log _{10} \Delta T_{\text {room }}$, and the difference of temperature rise between the fire room and the wall $\log _{10}\left(\Delta T_{\text {room }}-\Delta T_{\text {wall }}\right)$. Plots indicates the conversion times of nine panels at Side-B. It can be seen from Fig. 7 (2) that $\zeta$ is dependent on $C_{s}$.

As $\zeta$ obtained from ${ }_{a} \tau$ is a cumulative result after the transient exposure to smoke in this experiment, we perform the integration of $C_{s}$ for total exposure time $\left(\int C_{s} d t\right)$. The results at low temperature of fire room $\log _{10} \Delta T_{\text {room }}$ were affected by the position of 
panels, therefore we only treat the results at high temperature in the combustion as the data being valid ( $\Delta T_{\text {room }}-\Delta T_{\text {wall }} \geq 2.0 \mathrm{~K}$ ). In Fig. 8, the values of $\zeta$ of each fire source

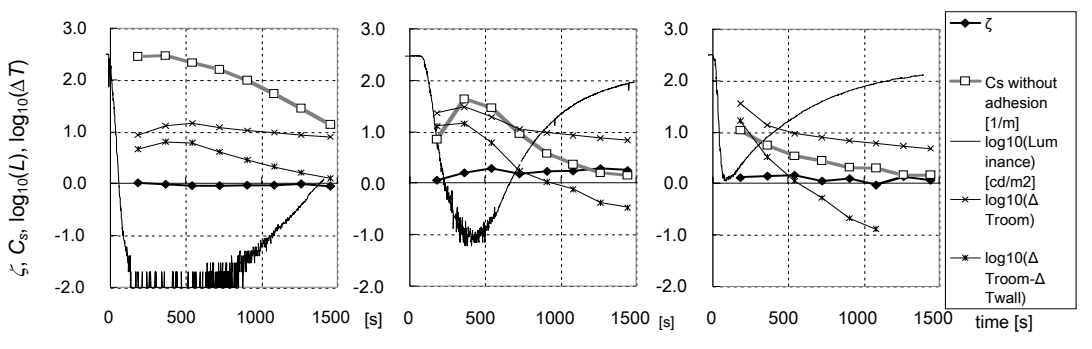

$\begin{array}{lll}\text { (1) Cotton } & \text { (2) Kerosene } & \text { (3) Soft-Polyurethane }\end{array}$

Fig. 7. Experimental results (natural vent condition).

are plotted to versus $\int C_{s} d t$ for each ventilation velocity. Both kerosene and softpolyurethane show linear correlation between $\zeta$ and $\int C_{s} d t$ with similar values of regression coefficient, while in the case of cotton $\zeta$ remains around 0 irrespective of $\int C_{s} d t$. The ventilation velocity may affect the relationship but the difference among the ventilation velocity is not so significant, as long as the present experiments are concerned. We obtained a common regression line for kerosene and soft-polyurethane because they tend to show similar correlations.

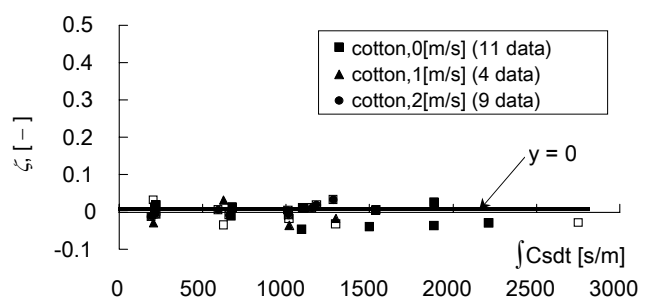

(1) Cotton

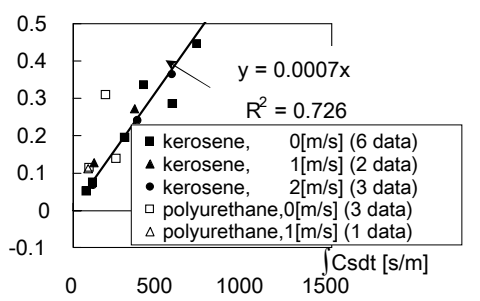

(2) Kerosene \& Soft-Polyurethane

Fig. 8. Relationship between $\zeta$ and $C_{s}$.

Therefore, the relation between $\zeta$ and $\int C_{s} d t$ is represented by

$\zeta=\eta \int C_{s} d t$

where the regression coefficient $\eta \mathrm{m} / \mathrm{s}$ is adhesion coefficient.

Adhesion coefficients of three fire sources are obtained from the above data irrespective of ventilation velocity as follows:

$\eta=$ const. $=\left\{\begin{array}{l}0.0007 \text { (Flaming Kerosene and Soft }- \text { Polyurethane }) \\ 0 \quad \text { (Smoldering Cotton) }\end{array}\right.$ 
In this paper, we only measured $\eta$ of three kinds of fire sources. However, the values of $\eta$ for different fire sources can be obtained easily by Eq. 17 if Eq. 15 holds for any fire source.

$\eta=\frac{\zeta}{\int C_{s} d t}$

\section{THE CALUCULATION MODEL OF LUMINANCE TAKING INTO ACCOUNT OF SMOKE ADHESION}

\section{Estimation of Object's Transmittance and Reflectance with Smoke Adhesion}

The adhered transmittance ${ }_{a} \tau$ as defined in this paper can be interpreted as the ratio of the non- adhered area to the total area of the object panel. It is considered that area with no smoke adhesion maintains the original reflectance. ${ }_{a} \tau$ can be estimated

$$
{ }_{a} \tau={ }_{0} \tau(1-\zeta)={ }_{0} \tau\left(1-\int \eta C_{s} d t\right)
$$

Letting $\rho_{b}$ be the reflectance of adhered smoke (virtually no reflection), reflectance decreased by smoke adhesion ${ }_{a} \rho$ can be estimated by

$$
{ }_{a} \rho \neq \rho_{\text {smoke }} \zeta+{ }_{0} \rho(1-\zeta)={ }_{0} \rho-\int \eta C_{s} d t\left({ }_{0} \rho-\rho_{\text {smoke }}\right)
$$

\section{The Calculation Model of Target Luminance in Evacuee's View Taking into Account of Smoke Adhesion}

We construct the calculation model [4] for target luminance based on Chandrasekhar [5] and Matsuura [6]. The concept of this calculation model is shown in Fig. 9. This model consists of three situations, i.e., (i) both evacuee's eye and a target are in upper smoke layer, (j) the both are in lower fresh-air layer, and (ij) the evacuee's eye and a target are in

\begin{tabular}{|c|c|}
\hline upper smoke layer & $\begin{array}{lll}\text { Factors of } & \text { Cs }[1 / \mathrm{m}], \mathrm{k}, & \rho_{i}, \mathrm{~A}_{\mathrm{i}}\left[\mathrm{m}^{2}\right] \\
\text { upper smoke } & \mathrm{F}_{\text {smoke }}[\mathrm{lm}] & \mathrm{A}_{\text {smokek }}\left[\mathrm{m}^{2}\right] \\
\text { layer } & \mathrm{L}_{\text {smoke }}\left[\mathrm{cd} / \mathrm{m}^{2}\right] & \text { (including } \mathrm{A}_{\text {interface }} \text { ) } \\
\end{array}$ \\
\hline $\mathrm{D}(\mathrm{i})+\mathrm{D}(\mathrm{j})[\mathrm{m}]$ & $\mathrm{A}_{\text {nterface }}\left[\mathrm{m}^{2}\right]$ \\
\hline $\begin{array}{c}\text { lower air } \\
\mathrm{D}(\mathrm{j})[\mathrm{m}]\end{array}$ & $\begin{array}{ll}\mathrm{F}_{\text {air }}[\mathrm{lm}] & \rho_{j}, \mathrm{~A}_{\mathrm{i}}\left[\mathrm{m}^{2}\right] \\
\mathrm{L}_{\text {air }}\left[\mathrm{cd} / \mathrm{m}^{2}\right] & \mathrm{A}_{\text {dir }}\left[\mathrm{m}^{2}\right] \\
& \text { (including } \mathrm{A}_{\text {interface }} \text { ) }\end{array}$ \\
\hline
\end{tabular}
different layer each other.

Fig. 9. Concept of the calculation model of target luminance in evacuee's view taking into account of smoke adhesion by two-layer zoom model.

The calculation model of luminance in evacuee's view is expressed by the set of Eq. 20, Eq. 21, and Eq. 22, which involve smoke density and smoke adhesion. 
Here, the intensity of surrounding light $\mathrm{F} \mathrm{lm}$, transmittance of emergency signs, and reflectance of wall change as adherence increases. For considering common emergency signs, we set up light source luminance $L_{e}$, transmittance of acrylic panel $\tau_{e}$, and surface reflectance of the panel $\rho_{e}$ in the equations (in Fig. 10). In addition, this model can also be applied to non-emissive visual objects like reflecting-typed signs.

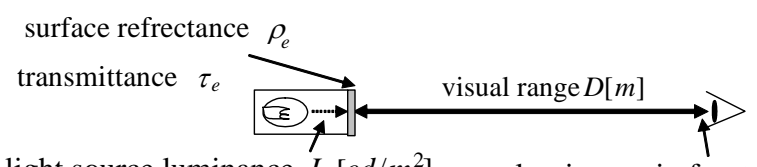

light source luminance $L_{e}\left[\mathrm{~cd} / \mathrm{m}^{2}\right] \quad$ luminance in front of observer's eye $L_{D}\left[\mathrm{~cd} / \mathrm{m}^{2}\right]$

Fig. 10. The target of the calculation model of target luminance in evacuee's view.

(i) Evacuee's eye and target in upper smoke layer

$$
\begin{aligned}
& { }_{a} L_{D(i)}=\left({ }_{a} \tau_{e} L_{e}+{ }_{a} \rho_{e} L_{\text {smoke }}\right) e^{-C_{s} D(i)}+L_{\text {smoke }} k\left(1-e^{-C_{s} D(i)}\right) \\
& \text { where }\left\{\begin{array}{l}
L_{\text {smoke }}=\frac{\sum_{a} \rho_{i} A_{i} \times \Sigma_{a} F_{\text {smoke }}}{\pi\left(A_{\text {smoke }}\right)^{2}}+\frac{A_{\text {interface }}}{A_{\text {smoke }}} \times \frac{\sum \rho_{j} A_{j} \Delta F_{\text {air }}}{\pi\left(A_{\text {air }}\right)^{2}} \\
{ }_{a} \rho_{e, i}=\left(1-\int \eta C_{s} d t\right) \tau_{e} \\
\sum_{a, i}-\int \eta C_{s} d t\left(\rho_{e, i}-\rho_{\text {smoke }}\right)
\end{array}\right\}
\end{aligned}
$$

(j) Evacuee’s eye and target in lower air layer

$L_{D(j)}=\tau_{e} L_{e}+\rho_{e} L_{a i r}$

where $L_{\text {air }}=\frac{\Sigma \rho_{j} A_{j} \Sigma F_{\text {air }}}{\pi\left(A_{\text {air }}\right)^{2}}+\frac{A_{\text {interface }}}{A_{\text {air }}} L_{\text {smoke }}$

(ij) Evacuee's eye in different layer against target

$$
\begin{aligned}
& { }_{a} L_{D(i j)}=\left({ }_{a} \tau_{e} L_{e}+{ }_{a} \rho_{e} L_{\text {smoke }}\right) e^{-C_{s} D(i)}+L_{\text {smoke }} k\left(1-e^{-C_{s} D(i)}\right) \\
& \text { where }\left\{\begin{array}{l}
L_{\text {smoke }}=\frac{\Sigma_{a} \rho_{i} A_{i} \times \Sigma_{a} F_{\text {smoke }}}{\pi\left(A_{\text {smoke }}\right)^{2}}+\frac{A_{\text {interface }}}{A_{\text {smoke }}} \times \frac{\Sigma \rho_{j} A_{j} \Sigma F_{\text {air }}}{\pi\left(A_{\text {air }}\right)^{2}} \\
\tau_{e}=\left(1-\int \eta C_{s} d t\right) \tau_{e} \\
\rho_{e, i}=\rho_{e, i}-\int \eta C_{s} d t\left(\rho_{e, i}-\rho_{\text {smoke }}\right) \\
\sum_{a} F_{\text {smoke }}=\left(1-\int \eta C_{s} d t\right) \Sigma F_{\text {smoke }}
\end{array}\right\}
\end{aligned}
$$

\section{EXAMPLE BY USING THE CALCULATION MODEL TAKING INTO ACCOUNT OF SMOKE ADHESION}

We calculate the change of emergency sign's luminance in the case of power outage in fire. The results are shown in Fig. 11. In this case, we assumed that $\rho_{b}=0.1$ for 
convenience. In Fig. 11, we compare the luminance taking into account of smoke adhesion ${ }_{a} L_{D}$ with the initial luminance ${ }_{0} L_{D}$. It is shown in Fig. 11 that, when whole room is exposed to smoke at constant density, the luminance in evacuee's view decreases more as smoke density is higher and adhesion is greater.

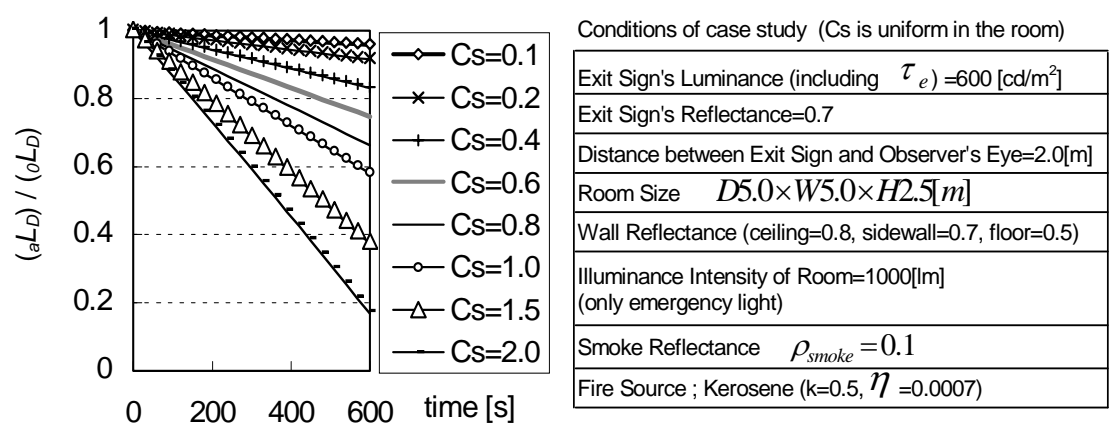

Fig. 11. Example by using of the model of target luminance in evacuee's view.

\section{CONCLUSION}

This paper proposed a calculation model to quantify the changes of visual stimulation in smoke of fire taking into account of smoke adhesion. By incorporating the effects of adhesion in the model, we can improve the estimation of luminance decay. It is considered to be useful to apply such a method to fire safety design of buildings for exploring effective exit sign planning addressed to safer evacuation in fire.

\section{ACKNOWLEDGMENT}

We would like to thank Prof. Taro Noguchi, Dr. Naoya Hara, Mr., Kentaro Tsuji (Kansai University) and Mr., Mitsuo Kadoya (Kansai Fire Protection Engineering Corporation) for valuable advice and cooperation.

\section{REFERENCES}

[1] Inoue, Y., and Akizuki, Y., "The Optimal Illuminance for Reading, Effects of Age and Visual Acuity on Legibility and Brightness,” Journal of Light \& Visual Environment, 22, No.1, pp. 23-33, 1998.

[2] Roufs, J.A., "Dynamic Properties of Vision-I. Experimental Relationships between Flicker and Flash Thresholds,” Vision Res., 12, p. 261, 1972.

[3] Akizuki, Y., and Inoue, Y., "The Concept of Visual Acuity Ratio to the Maximum Level of Individual Visual Acuity - The Evaluation Method of Background Luminance and Visual Distance on Visibility Taking into Account of Individual Visual Acuity," Journal of Light \& Visual Environment, 28, pp. 35-49, 2004.

[4] Akizukia, Y., and Tanaka, T., "Visibility of Text Information Functioning as Urban Disaster Prevention - The Visibility of Emergency Signs in the Daegu Subway Fire, Korea," Proceeding of 6th Asia-Oceania Symposium on Fire Science and Technology, Daegu, pp. 579-590, 2004.

[5] Chandrasekhar, S., Radiative Transfer, New York, Dover, 1960. 
[6] Matsuura, K., and Nakamura, Y., "Base Model of Optical Transmission Through Scattering Medium,” Summaries of Technical Papers of Annual Kinki Meeting Architectural Institute of Japan, pp. 37-44, 2003, (in Japanese).

[7] Jin, T., "Visibility Through Fire Smoke, Part 2. Visibility of Monochromatic Signs Through Fire Smoke," Report of Fire Research Institute of Japan, No. 33, pp. 31-48, 1971. 
